\title{
Philosophiques
}

\section{Les définitions aristotéliciennes de la justice : leurs rapports à la notion d'égalité}

\section{Jocelyne Saint-Arnaud}

Volume 11, numéro 1, avril 1984

URI : https://id.erudit.org/iderudit/203247ar

DOI : https://doi.org/10.7202/203247ar

Aller au sommaire du numéro

Éditeur(s)

Société de philosophie du Québec

ISSN

0316-2923 (imprimé)

1492-1391 (numérique)

Découvrir la revue

Citer cet article

Saint-Arnaud, J. (1984). Les définitions aristotéliciennes de la justice : leurs rapports à la notion d'égalité. Philosophiques, 11(1), 157-173.

https://doi.org/10.7202/203247ar
Résumé de l'article

$\mathrm{Au}$ livre $\mathrm{V}$ de l'Éthique à Nicomaque, Aristote ne donne pas une définition unifiée de la justice. Tout en ne rejetant pas la définition traditionnelle qui identifie la véritable justice et la justice légale, ni celle qui repose sur la justice naturelle et fonde la notion d'équité, il cherche de nouvelles assises pour la justice institutionnelle et c'est la notion d'égalité qui sert à cette fin. Cet article vise à préciser les notions de stricte égalité et d'égalité proportionnelle et corrélativement à analyser les définitions des justices distributive et directive qui constituent les branches de la justice particulière chez Aristote. 


\title{
LES DÉFINITIONS ARISTOTÉLICIENNES DE LA JUSTICE : LEURS RAPPORTS À LA NOTION D'ÉGALITÉ.*
}

\author{
par Jocelyne Saint-Arnaud
}

\begin{abstract}
RÉSUMÉ. Au livre $\mathrm{V}$ de l'Éthique à Nicomaque, Aristote ne donne pas une définition unifiée de la justice. Tout en ne rejetant pas la définition traditionnelle qui identifie la véritable justice et la justice légale, ni celle qui repose sur la justice naturelle et fonde la notion d'équité, il cherche de nouvelles assises pour la justice institutionnelle et c'est la notion d'égalité qui sert à cette fin. Cet article vise à préciser les notions de stricte égalité et d'égalité proportionnelle et corrélativement à analyser les définitions des justices distributive et directive qui constituent les branches de la justice particulière chez Aristote.
\end{abstract}

ABSTRACT. In the Fifth Book of the Nicomachean Ethics, Aristotle does not give one unified definition of justice. He does not reject the traditional definition of legal justice nor the definition of natural justice which is necessary to dread the idea of equity. However, he wants to ground institutional justice on a new base and the idea of equality serves this end. In this paper, I want to make clear the difference between strict and proportional equality and correlatively to analyse the definitions of distributive and "directive" justices which form the two branches of the aristotelian particular justice.

Avant Aristote, la notion de justice n'était comprise que par rapport à un droit d'origine divine. ${ }^{1}$ Il semble que l'avènement de la démocratie à Athènes ne soit pas étranger au fait que la justice se rationalise, le législateur formulant le nomos et

* Merci à André St-Arnaud, f.e.c. et aux membres de l'équipe, particulièrement à Louise Marcil-Lacoste et France Giroux, pour leur conseils judicieux.

1. Selon R.A. Gauthier et J.Y. Jolif dans l'Éthique à Nicomaque, tome II, Commentaire, Louvain, Publications Universitaires de Louvain, Paris, Éditions Béatrice-Nauwelaerts, 1959, p. 325 : la liaison des termes dikaios et boszos l'atteste chez Platon (Gorg. 507 b, Rep. 331 a, Eutbyphron 12 c-2). 
le juge rétablissant l'équilibre symbolisé par la balance. Pour le sens commun, la loi, non dépouillée de toute autorité divine, représente la justice et, dans la mesure où la Cité définit la totalité de l'existence humaine, la justice était devenue la vertu par excellence, la sauvegarde de l'ordre et de l'harmonie. Dire que «la justice contient toutes les autres vertus $»^{2}$, correspond donc à la notion communément admise en Grèce classique ; là où Aristote est novateur, c'est dans la systématisation qu'il a faite de la distinction entre la légalité et l'égalité comme fondement de la justice. Pourtant la philosophie aristotélicienne est reconnue pour être méritocratique, non pas égalitaire. Le but de cet article est de faire le point sur cette question à partir des définitions fournies par Aristote au livre $\mathrm{V}$ de l'Éthique à Nicomaque.

Ces définitions ne sont pas exposées de façon systématique et unifiée. Certains commentateurs, comme F. et C. Khodoss, R.A. Gauthier et J.Y. Jolif rétablissent un plan d'ensemble pour ce livre qui comporte des redites, des recoupements et même des contradictions. Quant à nous, nous préférons ne pas inférer au-delà du texte sauf pour ce qui concerne la base politique nécessaire à la compréhension de la justice distributive. À la -suite de_John_Burnet ${ }^{3}$ _et de _Léon Robin ${ }_{-}^{4}$, nous considérons_la politique, chez Aristote, comme la science architectonique dont dépend toute autre science pratique. L'homme, pour Aristote, est un être de Cité. Dans l'ordre des fins ou de la nature, "la Cité passe avant la famille et avant chacun de nous $»^{5}$. La nature d'une chose ou son essence étant son plein développement, le plein développement de l'homme et celui de la Cité coïncident, à savoir le bien vivre ou le bonheur.

Pour définir la justice au livre V de l'Étbique à Nicomaque, Aristote s'appuie sur les définitions courantes et utilise des méthodes variées : dissipation des équivoques reliées aux défi-

2. Aristote, Éthique à Nicomaque, trad. Jean Voilquin, Paris, Garnier, V, 1, 15 ; pagination Bekker 1130 a 10.

3. John Burnet, Aristotle on Educatton, being extracts from the Ethics and Politics translated by John Burnet, Cambridge, Cambridge University Press, 1931, 141 p.

4. Léon Robin, La pensée grecque et les origines de l'esprit scientifique, Paris, Albin Michel, 1963. À l'appui de cette thèse, il cite p. 311 note 1 : Eth. Nic. I, 1, 1094 a $24-\mathrm{b} 27 ; 13$, 1102 a 13-26 Pol. I, 1 in. ; 2, 1253 a 27.

5. Pol. I, 1,1252 b $27-1253$ a 39 . 
nitions courantes, recherche du juste milieu à partir des contraires ou des extrêmes, réponses à des objections, application des données abstraites à la vie pratique, etc. Il n'est donc pas étonnant qu'ainsi traitée, la justice supporte plusieurs définitions simultanément admises par Aristote. ${ }^{6}$

Mises à part les définitions qui traitent des conditions subjectives de l'acte juste dont nous écartons l'étude dans la présente analyse, les définitions de la justice au livre $\mathrm{V}$ de l'Éthique à Nicomaque s'élaborent par rapport à la légalité, à la nature, à l'équité et à l'égalité. Comme notre propos porte particulièrement sur les rapports de l'égalité et de la justice, nous ne présenterons que sommairement les définitions traditionnelles, afin d'étudier plus en détail celles qui reposent sur l'égalité et déterminent la justice particulière.

\section{DÉFINITIONS TRADITIONNELLES.}

\section{A. Justice et légalité.}

En tant qu'identique à la loi, la justice est la plus parfaite des vertus ; elle garantit l'ordre et l'harmonie au sein de la Cité.

On appelle juste en un premier sens, tout ce qui contribue à produire ou à entretenir pour une communauté politique le bonheur aussi bien dans sa totalité que dans les détails. ${ }^{7}$

Il s'agit ici d'un éloge des lois fondé sur les idées traditionnelles. Après Solon et Platon, Aristote reprend l'idée qu'il y a justice lorsque chacun accomplit sa tâche non uniquement pour son propre bien mais dans l'intérêt de la communauté ${ }^{8}$. En ce sens, la justice totalise toutes les vertus car elle traite du rapport de l'homme avec ses semblables et non du rapport de l'homme avec

6. Dans l'Éthique à Eudème (VII, 2.), Aristote est beaucoup plus explicite quant aux différentes définitions d'une même notion et à leur compatibilité. " Les positions contraires demeurent si ce qui est dit est vrai en un sens, mais non dans un autre " (VII, 2, $1235 \mathrm{~b} 17$ ). Il y établit qu'il y a trois sortes d'amitiés, "qu'elles ne se disent pas toutes selon un sens, ni comme les espèces d'un seul genre, ni d'une manière tout à fait équivoque " (VII, 2, 1236 a 15-17). « il est impossible de parler de toutes les amitiés selon une seule définition " (VII, 2, 1236 b 23). Aristote, Éthique à Eudème, Introduction, traduction, note et indices par Vianney Décaric, Paris/Montréal, Vrin/Presses de l'Université de Montréal, 1978.

7. Étbique à Nicomaque, traduction F. et C. Khodoss, Paris, Presses Universitaires de France, 1965, V, 2, 1129 b 17.

8. Platon, Rep. IV 433 a. 
lui-même comme c'est le cas des autres vertus. Que la justice légale (dikaiosunè) soit la représentation de la véritable justice, Aristote ne le nie pas. Il ne rejette pas la tradition mais il vit la décadence des cités libres auxquelles était si intimement liée cette conception de la loi. C'est pourquoi il cherchera de nouvelles assises pour la justice non pas dans le but d'en rejeter la base traditionnelle mais plutôt de la compléter et de la rendre plus effective.

\section{B. Justice et nature.}

Pour ce qui est de la justice naturelle, qui deviendra la base de la théorie du droit naturel, Aristote au livre $\mathrm{V}$ la tient pour acquise (1134 b 18) et il n'élabore pas particulièrement la distinction entre justice naturelle et justice légale. Il semble plutôt répondre à l'objection née probablement d'une meilleure connaissance des constitutions des autres contrées voulant que ces constitutions et lois ne puissent reposer sur une loi naturelle invariable du fait qu'elles varient en fonction du temps et de l'espace. Aristote argumente en ce sens : « que toutes les choses considérées comme justes soient variables, ne s'oppose pas à ce que certaines d'entre elles ne soient fondées sur la nature " ${ }^{9}$. Il est même facile selon lui de distinguer ce qui vient de la nature et ce qui vient de la convention ${ }^{10}$, comme le montrerait l'exemple de la possibilité pour toute personne de devenir ambidextre quoique, par nature, la main droite soit la plus forte.

Les prescriptions de la justice qui ne sont pas fondées sur la nature, mais sur les conventions entre les hommes, ne sont pas semblables partout, non plus que les formes de gouvernement, quoiqu'il n'y en ait qu'une seule qui se montre partout en accord avec la nature, à savoir la meilleure. ${ }^{11}$

Il s'agit évidemment de la conception aristocratique de l'État basée sur la vertu telle qu'élaborée par Aristote. Mais notre auteur ne précise pas ici en quoi une constitution est meilleure qu'une autre ni comment on peut vérifier son accord avec la nature.

9. R.A. Gauthier et J.Y. Jolif, op. cit. p. 394.

10. Eth. Nic. V, 10, 1143 b 30-35.

11. Eth. Nic, trad. Voilquin, V, 8, 5, p. $139 ; 1135$ a $1-5$. 


\section{Justice et équité.}

La justice comme équité, dont l'histoire a retenu la formule « à chacun selon son dû » comme étant caractéristique de l'analyse aristotélicienne de la justice, se rattache selon nous aux définitions traditionnelles parce qu'elle ne prend sens que par référence à la justice naturelle. En effet, au livre $\mathrm{V}$ de l'Étbique à Nicomaque, l'équité se présente comme une forme supérieure de justice rendue nécessaire par la généralité des lois qui doivent tenir compte des cas ordinaires et non de l'exception. La règle générale doit s'appliquer à tous les cas particuliers, il faut donc avoir recours à l'équité qui, par l'amendement, pallie aux insuffisances de la loi. Aristote compare l'équité à la règle de plomb dont se sont servis les constructeurs à Lesbos et qui, grâce à sa flexibilité, pouvait évaluer mieux qu'une mesure rigide la dimension des pierres nécessaires à la construction ${ }^{12}$. L'équité, ainsi entendue, ne s'oppose pas à la loi écrite comme c'est le cas dans la Rhétorique (I, 13) où elle signifie indulgence, mais se présente comme source du droit, loi inscrite au cœur des hommes (Dikè), conforme à la loi naturelle.

\section{DÉFINITIONS ARISTOTÉLICIENNES DE LA JUSTICE PARTICULIÈRE FONDÉES SUR LA NOTION D'ÉGALITÉ.}

Aristote est le premier à distinguer de façon systématique la légalité et l'égalité comme fondement de la justice, mais il n'est pas le premier philosophe à avoir défini l'égalité. Platon, quoique succinctement, avait soutenu dans les Lois VI 757 :

. . . il y a deux sortes d'égalités qui se ressemblent pour le nom, mais qui sont bien différentes pour la chose. L'une consiste dans le poids, le nombre, la mesure : il n'est point d'État, point de législateur, à qui il ne soit facile de la faire passer dans la distribution des honneurs, en les laissant à la disposition du sort. Mais il n'en est pas ainsi de la vraie et parfaite égalité, qu'il n'est point aisé à tout le monde de connaître : le discernement en appartient à Jupiter, et elle ne se trouve que bien peu entre les hommes. (. . . .) C'est elle qui donne plus à celui qui est plus grand, moins à celui qui est moindre, à l'un et à l'autre dans la mesure de sa nature; proportionnant ainsi les honneurs au mérite, elle donne les plus grands à ceux qui ont le plus de

12. Eth. Nic. V, 10, 1137 b 30. 
vertu, les moindres à ceux qui ont le moins de vertu et d'éducation, et à tous selon la raison. ${ }^{13}$

La première forme de distribution est égalitaire en ce qu'elle substitue au droit par privilèges (naissance, richesse, statut, compétence, etc. dirait-on aujourd'hui) une façon impartiale de partager les biens. Elle n'implique pas que le partage lui-même soit juste à moins que l'attribution ne soit spécifiée par d'autres règles. Cette pratique existait, par exemple, à Athènes sous Péricles. Le prytane épistate, celui qui représente la Cité, est tiré au sort quotidiennement et un citoyen ne peut assumer ce poste qu'une fois dans sa vie. ${ }^{14}$ Cette dernière spécification rend le partage égalitaire. Quant à la deuxième forme de distribution, c'est celle qu'Aristote a reprise en justice distributive sous la notion d'égalité proportionnelle.

Chez Aristote, la justice particulière se fonde sur la notion d'égalité. Selon les commentateurs Gauthier et Jolif, tout le livre $\mathrm{V}$ est bâti sur la distinction entre deux sens du mot dikaion qui peut signifier ce qui est légal et ce qui est égal. ${ }^{15}$

Examinons en combien de sens peut se prendre le mot injuste.

On appelle ainsi celui qui viole les lois et aussi celui qui prend plus que sa part et qui méconnaît l'égalité ; il est donc clair que -sera-juste celui' qui observe l'égalité- ; par conséquent-le juste sera légalité et égalité ; l'injuste, illégalité et inégalité. ${ }^{16}$

Après avoir défini le juste comme respect des lois, ce qui est admis et véhiculé par la tradition, Aristote doit démontrer comment le juste coïncide avec l'égal. Pour ce faire, il joint à la méthode de dissipation des équivoques (en l'occurence l'homonymie dikaion) celle de l'induction par l'explicitation de la notion contraire (nous avons un exemple de cette façon de procéder à la citation précédente où le juste est défini à partir d'une définition de l'injuste). Pour Aristote, non seulement les contraires peuvent-ils s'éclairer l'un l'autre, mais encore si des deux contraires, l'un se dit en plusieurs sens, il en sera de même pour l'autre.

13. Platon, Les Lois, traduction par Victor Cousin, Oeuvres de Platon, tome septième, Paris, Pichons et Didier, Librairies, 1931, p. 317.

14. François Châtelet, Les Idéologies, tome I, Paris, Hachette, 1978, p. 130.

15. R.A. Gauthier et J.Y. Jolif, op. cit. p. 323.

16. Eth. Nic, trad. F. et C. khodoss, V, 2, 1129 a 31-34. 
L'analyse faite par Aristote de l'homonymie dikaion avait permis de distinguer deux couples de contraires, légal-illégal et égal-inégal, pour la définition du juste. Après avoir défini le juste légal en passant par son contraire l'illégal, il conserve l'inégal pour la définition de la justice particulière ce qui lui permettra, par le jeu des contraires, de parvenir à l'appréhension de l'égal et par le fait même du juste. De cette façon, il parvient à définir l'égal comme la juste mesure entre les inégalités et le juste comme se situant au centre des extrêmes représentés par l'injuste.

Puisque l'injuste ne respecte pas l'égalité et que l'injustice se confond avec l'inégalité, il est évident qu'il existe une juste mesure relativement à l'inégalité. Cette juste moyenne c'est l'égalité. Dans les actes qui comportent le plus et le moins, il y a place pour une juste moyenne. Si donc l'injuste c'est l'inégal, le juste est l'égal. (. . .) Or puisque l'égal consiste dans une juste moyenne, il pourra en être ainsi du juste. ${ }^{17}$

Il apparaît évident que la théorie du juste milieu est adaptée ici aux besoins de la cause. Aristote ne peut traiter de la justice à la manière des autres vertus comme un juste milieu entre l'excès et le manque c'est pourquoi il établit ici le juste milieu en fonction de l'égalité, notion qui se prête bien à une explication à caractère géométrique et dont la signification se rapproche par bien des aspects de la notion même de juste milieu. On pourrait même penser que c'est en raison de ces difficultés qu'Aristote a défini la justice particulière en rapport avec l'égalité.

La justice particulière se distingue de la justice légale comme la partie du tout. Elle comprend la justice distributive qui concerne la " répartition des honneurs, des richesses, ou de tous les autres avantages qui peuvent échoir aux membres de la cité ${ }^{18}$ et la justice directive qui traite des échanges entre particuliers. La justice directive se divise en justice commutative qui traite des échanges librement consentis comme " la vente, l'achat, le prêt à intérêts, la caution, la location, le dépôt, le salaire ${ }^{19}$ et en justice corrective qui traite des rapports imposés. La justice corrective s'applique aux rapports imposés clandestins

17. Eth. Nic. trad. Voilquin, V, 3, 1-4; 1131 a $10-15$.

18. Eth. Nic, trad. Voilquin, V, 2, 12-13;1130 b 301131 a 8.

19. Ibid. 
comme « le vol, l'adultère, l'empoisonnement, la prostitution, le détournement d'esclave, le meurtre par ruse, le faux témoignage $»^{20}$ et aux actes de violence comme les « coups et blessures, l'emprisonnement, le meurtre, le pillage, la mutilation, la diffamation, l'outrage $»{ }^{21}$. Il est à remarquer que le meurtre peut être classé différemment selon l'intention du coupable.

Les termes employés pour désigner les subdivisions de la justice particulière proviennent de la traduction des commentateurs scholastiques. ${ }^{22}$ Il nous semble que seule cette traduction rend vraiment compte de la classification opérée par Aristote de 1130 b 30 à 1131 a 10 . Elle nous a permis de construire le tableau synoptique suivant $:^{23}$

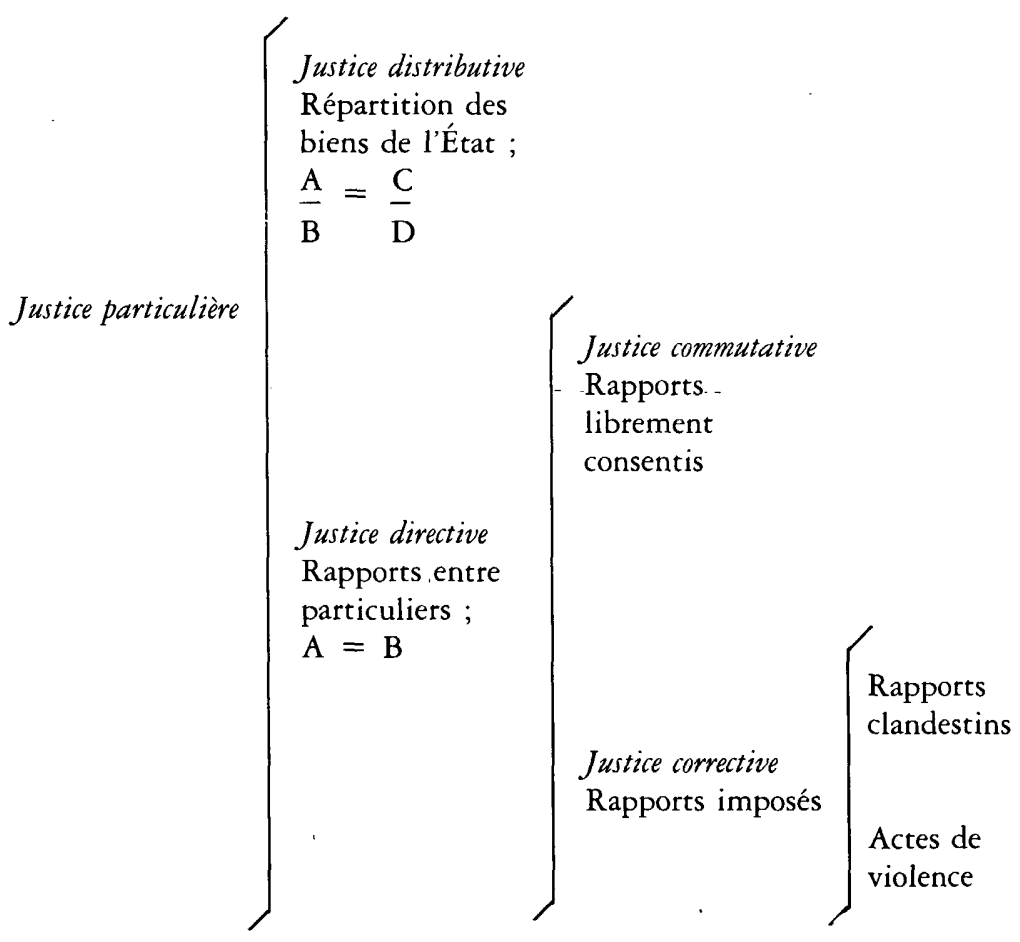

20. Ibid.

21. Ibid.

22. Selon John Burnet, la traduction scholastique est la meilleure. Voir son introduction au livre V The Etbics of Aristotle, Edited with and Introduction and Notes by John Burnet, Arno Press, New York, 1973, p. 203.

23. Pour la correspondance grecque de ce tableau, voir Henry Jackson, The Fifth Book of the Nicomachean Ethics of Aristotle, Arno Press, New York, 1973, p. 75. 


\section{A. Justice distributive}

Pour ce qui est de la justice distributive, le partage à effectuer doit être proportionnel au mérite ; il s'agit d'une égalité de rapport.

Il en sera de même de l'égalité, si l'on examine les personnes et les choses. Le rapport qui existe entre les objets se retrouvera entre les personnes. Si les personnes ne sont pas égales, elles n'obtiendront pas dans la façon dont elles seront traitées, l'égalité. De là viennent les disputes et les contestations, quand des personnes sur le pied d'inégalité, ont et obtiennent un traitement égal. Ajoutons que la chose est claire si l'on envisage l'ordre du mérite des parties prenantes. En ce qui concerne les partages, tout le monde est d'avis qu'ils doivent se faire selon le mérite de chacun ; toutefois, on ne s'accorde pas communément sur la nature de ce mérite, les démocrates le plaçant dans la liberté, les oligarques dans la richesse ou la naissance, les aristocrates dans la vertu. ${ }^{24}$

«Si les personnes ne sont pas égales, elles n'obtiendront pas dans la façon dont elles seront traitées, l'égalité ». ${ }^{25}$ Que signifie cette assertion ? Sur quoi repose le mérite ? Pour répondre à ces questions, nous nous référons à la Politique.

Pour Aristote, on l'a déjà mentionné, la Cité est primordiale et l'homme est un être de Cité. Dans la mesure où il accomplit sa fonction, il réalise le bien de l'ensemble. Aristote compare les citoyens aux matelots sur un navire :

... il est clair que les tâches de chacun font sa vertu propre, mais il y en a constamment une qui leur est commune à tous vu que tous ont pour but la sûreté de la navigation à laquelle ils aspirent et concourent, chacun à sa manière. De même, quoique les fonctions des citoyens soient dissemblables, tous travaillent à la conservation de leur communauté, c'est-à-dire au salut de l'État. C'est par conséquent, à cet intérêt commun que doit se rapporter la vertu de citoyen. ${ }^{26}$

On pourrait croire que vertu éthique et vertu civique coïncident, il n'en est rien sauf pour ceux qui sont à la tête de l'État. Seuls

24. Eth. Nic. trad. Voilquin, V, $3 ; 1131$ a $20-30$

25. Ibid.

26. Aristote, La Politique, texte présenté et annoté par Marcel Prélot, Paris, Denoel/Gonthier, 1980 , p. $49 ; 1276$ b $20-36$. 
les dirigeants réunissent en une même personne les vertus qui font l'homme de bien et le bon citoyen. ${ }^{27}$ Tous les citoyens sont tenus à la vertu civique, seuls les dirigeants sont tenus à la vertu éthique. La vertu civique vise le bien commun, la vertu éthique repose non seulement sur le degré de participation à la vie politique mais aussi sur les qualités morales intrinsèques qui permettent cette participation.

En tant qu'ils prennent part aux suffrages et aux assemblées, les citoyens sont également libres, mais en tant que certains doivent diriger d'autres obéir, ils ne possèdent pas la vertu éthique au même titre. L'aristocrate possède la vertu en totalité, les autres n'en possèdent que ce qui leur est nécessaire pour remplir leur fonction.

Cette théorie de la vertu éthique ou mérite s'appuie sur la croyance que l'homme se compose d'un corps et d'une âme ; l'âme commande par nature, le corps obéit. Par analogie, Aristote soutient qu'il existe des êtres supérieurs par nature qui sont faits pour commander et des êtres inférieurs par nature qui sont faits pour obéir. Ces êtres ne possèdent pas les mêmes qualités de l'âme :

bien que les parties de l'âme soient présentes en tous ces êtres, elles y sont cependant présentes d'une manière différente : l'esclave est totalement privé de la partie délibérative ; la femelle la possède mais démunie d'autorité ; quant à l'enfant, il la possède bien, mais elle n'est pas développée. Nous devons donc nécessairement supposer qu'il en est de même en ce qui concerne les vertus morales : tous doivent y avoir part, mais non de la même manière, chacun la possède seulement dans la mesure exigée pour remplir la tâche qui lui est personnellement assignée. ${ }^{28}$

Les êtres sont donc hiérarchisés naturellement, ils ne possèdent pas le même degré de mérite.

Ainsi, si l'on revient à l'étude du livre $\mathrm{V}$ de l'Éthique à Nicomaque, les personnes de même mérite, c'est-à-dire celles qui possèdent la vertu morale à un même degré, recevront un traitement égal, les autres un traitement différent proportionnel à

27. Idem, p. $50 ; 1293$ b 7. Il s'agit ici du régime aristocratique.

28. Aristote, La Politique, introduction, notes et index par J. Tricot, Paris, Vrin, 1977, I, 13,1260 a $10-24$. 
leur degré de mérite. Ainsi, si $\mathrm{A}$ et $\mathrm{B}$ sont des citoyens et $\mathrm{C}$ et $D$ des biens à partager, la justice distributive exige que $\frac{A}{B}=\frac{C}{D}$ ou que $\frac{A+C}{B+D}=\frac{A}{B}$. Il ne s'agit évidemment pas d'une stricte égalité mais dune égalité de rapport, ce qu'Euclide nomme proportion géométrique. Non seulement il ne s'agit pas ici d'une stricte égalité mais encore doit-on supposer $A \neq B$ pour que l'équation soit effective.

En résumé, le partage des biens et des honneurs régi par la justice distributive n'est pas un partage égalitaire, il est proportionnel au mérite. Du point de vue de l'ensemble des individus qui composent la société, la justice distributive ne présume pas leur égalité mais leur degré de vertu. Seuls sont tenus pour égaux les citoyens qui possèdent le même niveau de mérite d'où l'expression : "l'égalité aux égaux ».

\section{B. Justice directive}

Si la justice distributive se définit comme la répartition des honneurs et des biens en fonction d'une égalité proportionnelle fondée sur le mérite, la justice directive, elle, intervient dans les transactions, rapports ou échanges tant libres qu'imposés en fonction d'une égalité arithmétique $(A=B)$. Il s'agit encore là de rétablir la juste proportion ou le juste milieu. Mais cette justice est plus conventionnelle :

. . . il importe peu que ce soit ún homme de bien qui ait dépouillé un misérable ou réciproquement ; que ce soit un homme de bien ou un misérable qui ait commis l'adultère; la loi n'a d'égard qu'à la différence des torts et considère les parties comme égales. ${ }^{29}$

La loi ne considère que les torts et rétablit l'égalité de sorte que les « parties n'augmentent ni ne diminuent ce qu'elles possèdent, mais elles ont exactement la même chose qu'avant ". ${ }^{30}$ On dit alors que chacun a sa part sans gain ni perte. Rétablir l'égalité, c'est maintenir ce qui existait avant l'offense.

Cette définition fait problème car elle ne s'applique vraiment qu'aux rapports ou échanges librement consentis. Le langage employé par Aristote pour décrire le juste correctif est celui

29. Eth. Nic. trad. F. et C. Khodoss, V, $7 ; 1131$ b 25 - 1132 a 13.

30. Idem, 1132 b $11-20$. 
du juste commutatif (gain et perte) et ceci pose des problèmes dont Aristote est conscient :

On emploie communément ces mots et dans un sens général dans les cas de cette nature, bien que l'expression ne semble pas convenir à certains d'entre eux : par exemple, on parle du profit de celui qui frappe et de la perte de celui qui est frappé. ${ }^{31}$

En fait, le problème s'applique à tout le domaine du correctif autre que le vol. Comment rétablir les choses telles qu'elles étaient avant l'offense dans le cas du meurtre, par exemple, où il n'y a pas réciprocité entre le gain du coupable et la perte de la victime ? Aristote solutionne le problème en ayant recours au juge :

... . mais quand le juge a évalué le mauvais traitement, l'un devient le perdant, l'autre le gagnant. Ainsi l'égal est-il le juste milieu entre le plus et le moins ; le gain se confond avec le plus ; la perte, au contraire, avec le moins . . . 32

Ainsi, le juge est l'homme du juste milieu, il maintient la balance égale entre les deux parties.

Prenons une comparaison : Une ligne ayant été coupée en deux parties inégales, le juge prend ce qui, dans la partie la plus grande, dépasse la moitié et ce qui est repris ainsi est ajouté à la partie la 'plus petite:-Quant le tout'est partagé également, chacun reconnaît avoir ce qui lui revient ; des deux côtés les parties sont égales. ${ }^{33}$

Aristote, comme dans le cas de la justice distributive, nous amène à un plus haut degré d'abstraction et nous propose finalement l'explication géométrique qui permet la découverte du juste milieu et la fonde.

Supposons trois lignes AA, BB, CC, égales entre elles. Enlevons à $\mathrm{AA}$ la partie $\mathrm{AE}$ et ajoutons cette fraction $\mathrm{CD}$ à $\mathrm{CC}$. Ainsi toute la ligne $C C D$ surpasse la ligne $A E$ des parties $C D$ et $C F .{ }^{34}$

Pour rétablir l'égalité, il faudra rendre $C D$ à $A E$.

Le recours au jugement d'un médiateur, le juge, ne solutionne notre problème qu'à demi. En effet, l'impartialité du

31. Eth. Nic. trad. Voilquin, V, 4, $5 ; 1132$ a 10-14.

32. Idem, V, 4, 6; 1132 a 20 .

33. Idem, V, 4,$8 ; 1132$ a 25 .

34. Idem, V, 4, $12 ; 1132$ b $6-11$. 
juge implique qu'il considère les parties comme égales et si le schème du juste milieu pouvait s'appliquer à tous les cas qui concernent la justice directive tant commutative que corrective, l'explication serait acceptable. Mais comme nous l'avons souligné plus haut, le schème ne s'applique que très partiellement à la justice corrective. De plus, dès que le juge prononce sa sentence et qu'il décide d'un coupable, il ne considère plus les parties comme égales. Enfin, dans les faits, la peine imposée par le juge n'est pas égale au tort commis ; c'est pourquoi Aristote nous met en garde contre l'idée de relier la justice directive et la loi du talion, loi qui implique, d'une certaine façon, égalité et réciprocité entre le tort commis et la peine imposée. ${ }^{35}$

Par la peine imposée, le juge compense pour la perte encourue par la victime et c'est par cet aspect que la justice corrective se rattache à la justice directive. Mais ce qu'Aristote n'a point vu, dû à sa division de la justice en justice distributive et directive selon qu'il s'agit du partage des biens de l'État ou d'échanges entre particuliers, c'est que la justice corrective, mis à part le point de vue purement formel de l'égalité devant la loi, n'est pas une justice fondée sur l'égalité arithmétique à cause justement de la nature de la sentence. Dans les faits, le juge ne rétablit pas l'égalité, il impose une peine proportionnelle au tort commis en fonction d'une échelle de valeurs variable selon le temps et le lieu. Nous devons conclure que les problèmes suscités au niveau de la justice directive par l'utilisation d'un langage propre au domaine des échanges ne représentent que la face visible d'un problème plus fondamental : la justice corrective aurait pu être expliquée adéquatement à partir du schème développé en justice distributive ; elle ne correspond pas comme le pense Aristote à une égalité arithmétique mais à une égalité proportionnelle.

Le recours au juge n'en comporte pas moins un aspect positif que nous allons maintenant évaluer. Il est significatif qu'Aristote utilise le schème explicateur de la stricte égalité ou égalité arithmétique pour expliquer le type de rapport qui régit les échanges librement consentis (modèle contractuel). Ces échanges supposent une entente à l'intérieur de laquelle chacune des par-

35. Les pythagoriciens définissaient la loi du talion : ce qu'on fait subir à autrui après l'avoir subi soi-même. Eth. Nic. trad. Voilquin, V, 5, $1 ; 1132$ b 25. 
ties trouve son dû (équité) et qu'elles s'entendent également à respecter (sujétion à une règle établie de plein gré). La philosophie contemporaine utilise encore le contrat comme modèle de rapport humain égalitaire.

Par ailleurs, le recours à un tiers par les contractants apporte une garantie sociale nécessaire à la protection des individus en cause dans le cas où l'entente ne serait pas respectée. Ainsi en est-il du recours au juge en justice commutative.

Dans la personne du juge on cherche un tiers impartial et quelquesuns appellent les juges des arbitres ou des médiateurs, voulant signifier par là que, quand on aura trouvé l'homme du juste milieu, on parviendra à obtenir justice. ${ }^{36}$

C'est par l'intermédiaire du juge qu'Aristote introduit l'égalité devant la loi qu'il exprime de la façon suivante :

. . . peu importe que ce soit un homme distingué qui ait dépouillé un homme de rien, ou réciproquement ; (. . .) la loi n'envisage que la nature de la faute, sans égard pour les personnes qu'elle met sur un pied d'égalité. Il importe peu que ce soit un tel ou un tel qui commette l'injustice ou qui la subisse, un tel ou un tel qui cause le dommage ou en soit victime. ${ }^{37}$

Envisagée sous cet aspect, la loi confere l'égalité. Il s'agit d'une norme devant laquelle tous les membres de la Cité s'inclinent dans l'espoir qu'elle les protège et qu'ils pourront d'une façon ou d'une autre s'en prévaloir. ${ }^{38}$

Historiquement, cette question de droit est basée sur une question du fait. L'égalité devant la loi en Grèce provient des réformes de Dracon, de Solon et de Clisthènes. Or François Châtelet écrit qu'au VII ${ }^{\mathrm{e}}$ siècle, la lutte est si forte entre les familles féodales qui se battent pour la primauté politique et religieuse que les diverses parties en lutte font appel à l'arbitrage d'un homme, le nomothète, choisi pour son honnêteté, afin qu'il édicte des règles imprescriptibles qui vont à l'avenir servir au règlement des différends. Ainsi, le

36. Idem, $\mathrm{V}, 4,7 ; 1132$ a 20.

37. Idem, V, 4,$3 ; 1132$ a $1-6$.

38. Henri Buch, La notion d'égalité dans les principes généraux du droit, Revue Internationale de Philosophie, v. 25, 1971, p. 351-380. 
pouvoir traditionnel est limité et contesté du seul fait qu'il est soumis à plus puissant que lui - la loi - et qu'il est tenu de rendre raison publiquement de la décision judiciaire et de la sentence. En cela consiste la révolution draconienne : non seulement imposer un «c'est comme ça » valable pour tous égalisant ainsi sous un certain aspect, le statut de chacun au sein de la communauté —, mais surtout exiger la publicité du jugement. ${ }^{39}$

Ce qui est particulièrement intểressant dans cette question, c'est qu'à une époque peu éloignée de l'origine des cités, des lois et surtout des premières constitutions, un philosophe témoigne de l'aspect purement conventionnel de l'égalité. Elle ne se fonde pas dans la nature comme chez Hobbes mais provient d'un rapport établi par l'homme pour régir les échanges et les différends.

L'égalité devant la loi se présente comme condition minimale pour l'établissement de la justice institutionnelle mais elle n'implique pas que la loi elle-même soit juste. C'est pourquoi aujourd'hui, on distingue justice formelle : «principe d'action selon lequel les êtres d'une même catégorie essentielle seront traités de la même façon ${ }^{40}$ et justice matérielle qui spécifie la caractéristique essentielle à la formation des catégories que ce soit le besoin, le mérite, la naissance, la richesse . . . Du point de vue formel, plus la classe formée est vaste, plus l'application de la norme ou de la loi est égalitaire (par exemple, loi du suffrage universel). Aristote, dans la Politique, a bien vu que la démocratie est la forme de gouvernement la plus égalitaire mais pour lui elle n'est pas la plus juste parce que son critère d'évaluation de la justice distributive repose matériellement sur le mérite.

En conclusion, le livre V de l'Éthique à Nicomaque discute d'abord de définitions qui proviennent de la tradition commune et philosophique. Mais ces définitions, qui avaient accompagné l'âge d'or des Cités grecques au cours duquel était apparue la démocratie à Athènes, s'avèrent inopérantés au moment où s'édifient les philosophies platonicienne et aristotélicienne. La faillite des Cités est associée évidemment à plusieurs causes : guerres

39. François Châtelet, op. cit. p. 127.

40. Chaïm Perelman, Justice et Raison, Bruxelles, Éditions de l'Université de Bruxelles, 1972, p. 26. 
nombreuses, spécialisation des producteurs qui ont trouvé de nouveaux marchés, créations de monopoles, spéculation . . . ${ }^{41}$ mais une des causes est probablement rattachée par les philosophes à l'établissement de la démocratie. ${ }^{42}$ Le pouvoir du plus grand nombre ${ }^{43}$ (les esclaves ne sont pas des citoyens) est accusé d'amener le désordre dans la Cité en permettant à des démagogues de fonder le pouvoir sur des décrets plutôt que sur la loi, à des opportunistes de satisfaire leur intérêt personnel, allant ainsi à l'encontre du bien de l'État. La condition du rétablissement de l'ordre dépend pour Aristote de la pratique de la vertu. Si les hommes sont vertueux, surtout les dirigeants, on verra renaître l'ordre et l'harmonie au sein de la Cité d'où le critère de justice distributive fondé sur le mérite du plus vertueux. Chez Aristote, comme chez Platon, le pouvoir doit appartenir à l'élite. L'élitisme se définit cette fois non seulement par rapport au savoir mais par rapport à la vertu.

Comme critère de justice matérielle, la vertu éthique ou le mérite opère une hiérarchisation des individus en fonction de qualités qui sont intrinsèques à l'âme humaine. Le degré de vertu ne dépend pas uniquement de l'apprentissage qu'une éducation adéquate pourrait rendre accessible à tous mais se fonde sur un ordre établi par la nature. En-fonction-de cet ordre, les dirigeants sont des êtres parfaits, ils possèdent la vertu éthique en totalité et de ce fait, ils méritent plus de biens et d'honneurs. La justice distributive chez Aristote est donc fondamentalement inégalitaire.

Quant à la justice directive, celle qui concerne les échanges entre particuliers, elle repose sur une égalité formelle qui se

41. J.P. Vernant, Les Origines de la Pensée Grecque, Paris, Presses Universitaires de France, 1962, p. 61-73.

42. Aristote dans la Politique ne compte pas au nombre des citoyens ceux qui ont été naturalisés par Clisthènes. De plus, il condamne toute forme de démocratie où la loi ne serait pas souveraine (Pol: IV. 3, 1290 a 30-1290 b 20). Il craint le pouvoir de la masse (voir note 43) et surtout des démagogues. Tous réunis en masse ont un sentiment du juste, mais chaque individu pris à part est fort incapable de juger avec discernement (Pol. III, 6, 1281 b $23-1282$ a 41 ).

43. Il s'agit évidemment des citoyens ; c'est le gouvernement du plus grand nombre par rapport à la monarchie et à l'aristocratie. "On estime de manière approximative que, sous Péricles, Athènes pouvait avoir de 300,000 à 400,000 habitants, soit : environ 40,000 citoyens, environ 80,000 métèques, le reste étant constitué par des étrangers et des esclaves " (J. Maillet, Institutions et faits sociaux, Sirey, 1957, p. 91, note 1). 
réalise concrètement dans ce qu'il convient d'appeler l'égalité devant la loi. Cette égalité est procédurale mais il ne faut pas négliger son importance car elle est une condition logique essentielle pour l'institutionnalisation de la justice. Soulignons de plus qu'Aristote a laissé entrevoir, par sa classification des échanges librement consentis en justice directive, comment la formule contractuelle pouvait devenir le modèle paradigmatique des rapports humains égalitaires, ce qui sera élaboré entre autres par J.-J. Rousseau et John Rawls.

Enfin, si nous mettons de côté le critère de justice matérielle qui varie historiquement et que nous nous en tenions aux rapports strictement formels, Aristote, par la systématisation qu'il a faite du recours à l'égalité géométrique et arithmétique pour la solution des problèmes de justice distributive et directive, a fourni les schèmes explicateurs de tout partage de biens et de pouvoirs : l'un est proportionnel $\left(\frac{A}{B}=\frac{C}{D}\right)$, l'autre est égalitaire $(\mathrm{A}=\mathrm{B})$. Lequel est le plus juste ? Aristote répondrait : "Les deux sont justes ; l'un traite du partage des biens de l'État, l'autre des échanges entre particuliers ". Mais il faut admettre que l'égalité proportionnelle implique l'égalité arithmétique en autant qu'elle détermine des classes à l'intérieur desquelles les parties sont égales en fonction d'une caractéristique commune (même degré de mérite). Formellement parlant, l'égalité arithmétique est la formule la plus égalitaire et la plus juste. Appliquée universellement, elle supposerait l'existence d'une seule classe d'individus et un traitement égal pour tous.

Département de philosophie

Université de Montréal 